\title{
Cycloplegic Refraction in Children: A Complete Audit Cycle
}

\author{
Suma Ganesh ${ }^{*}$, Priyanka Arora², Sumita Sethi ${ }^{3}$, Chandra Gurung ${ }^{4}$ \\ ${ }^{1}$ Department of Pediatric Ophthalmology and Strabismus, Dr Shroff's Charity Eye Hospital, New Delhi, India \\ ${ }^{2}$ Department of Ophthalmology, Dayanand Medical College and Hospital, Ludhiana, India \\ ${ }^{3}$ Department of Ophthalmology, BPS Government Medical College for Women, Sonepat, India \\ ${ }^{4}$ Department of Ophthalmology, NNJS Banke Fateh Bal Eye Hospital, Nepalganj, Nepal \\ Email: *drsumaganesh@yahoo.com
}

Received 17 December 2014; accepted 14 February 2015; published 15 February 2015

Copyright (C) 2015 by authors and Scientific Research Publishing Inc.

This work is licensed under the Creative Commons Attribution International License (CC BY).

http://creativecommons.org/licenses/by/4.0/

c) (7) Open Access

\section{Abstract}

Purpose: Use of appropriate cycloplegic agent is an essential area of management in children with strabismus and refractive error. This study was designed to audit our own department's understanding and practice with respect to cycloplegia. Methods: Children in age group of 0 - 12 years with refractive errors and strabismus were evaluated with respect to four parameters: adherence to cycloplegic refraction (group-I), choice of cycloplegic agent (group-II), dosage of cycloplegia (group-III) and duration of cycloplegia (group-IV). Following the initial audit, the hospital audit committee evaluated the results; thereafter concerned staff was educated and aide-memoires of the dilatation protocol were introduced; a second audit cycle was carried out after 3 months. Results: First and second audit cycle included 334 children (mean age $6.2 \pm 2.2$ years) and 436 children (mean age $7.25 \pm 2.9$ years) respectively. A statistically significant improvement was found in all four parameters in the second audit cycle: adherence to dilation protocol $(82.3 \%$ in first cycle to $94.3 \%$ in second cycle; $p=0.001)$, choice of cycloplegic agent $(77 \%$ in the first cycle to $94.8 \%$ in the second cycle; $p=0.001)$, dosage of cycloplegic agent $(84 \%$ in the first cycle to $96.3 \%$ in the second cycle; $p=0.001)$ and duration of cycloplegic agents $(65 \%$ in the first cycle to $97.5 \%$ in the second cycle; $p=0.001$ for CTC and $71.8 \%$ in the first cycle to $98 \%$ in the second cycle; $p=0.001$ for Tropicamide). Conclusions: A complete audit cycle demonstrated a statistically significant improvement in all four parameters related to cycloplegic refraction in children. Regular auditing coupled with targeted interventions aimed to maintain the "best practice guidelines" for determination of refractive errors in children could prove effective in improving standards of clinical practice.

${ }^{*}$ Corresponding author. 


\section{Keywords}

\section{Cycloplegic Refraction, Audit, Children}

\section{Introduction}

Cycloplegic refraction has been described as an essential part of the paediatric ophthalmic assessment [1] and the cornerstone of strabismus evaluation [2]. An ideal cycloplegic agent removes the detrimental effects of accommodation on measurement repeatability thus revealing the correct and appropriate refractive error. The primary differences in the action of the various cycloplegics used in the clinical practice are the time course for the onset and recovery of cycloplegia and the depth of cycloplegia. In order to obtain reliable measurements following instillation of a particular cycloplegic drug, there is a time limit for maximum cycloplegia to be reached and for refraction to be carried out. However overmedication, when maximum cycloplegia has already been reached increases the probability of systemic absorption and therefore intensifies the side effects. The use of appropriate cycloplegic agent in appropriate dosage and for appropriate duration, for different categories of children with strabismus and refractive error is thus an important and a challenging area of paediatric ophthalmology.

Refractive errors and strabismus are the major causes of ocular morbidity amongst children and appropriate refractive correction is vital to attain optimal vision and binocularity. However in literature, there is no specific mention regarding evidence based standards of refraction practices in children. In order to maintain the "best practice" guidelines for determination of refractive errors according to different categories of patients, we planned a complete audit cycle at our centre with the following aims:

1) To evaluate our own department's understanding and practice with respect to cycloplegia.

2) To ensure that all optometrists and doctors in the practice team carry out examinations to the same standard; so as to improve clinical effectiveness.

\section{Materials and Methods}

The study was planned and carried out at the department of Paediatric Ophthalmology and Strabismology of Dr. Shroff's charitable eye hospital after approval from the institutional review board. A full audit cycle was planned in 2 cycles; in the first cycle data was collected over a 4-week period, following which a 3 month period was allowed to incorporate implemented changes and a second audit cycle was planned.

1) Subjects: All children younger than 12 years of age requiring cycloplegic refraction for refractive error or strabismus were included in the study after taking informed consent from parents. Patients were excluded if they were emmetropic or were older than 12 years of age.

2) Methodology: It has been our department's policy to place stickers for details of cycloplegic refraction on all the case sheets of children younger than 12 years of age; notes were audited using these stickers. Variables collected included the age of the patient, type of refractive error (myopia/hyperopia), presence or absence of strabismus, type of strabismus (esotropia/exotropia), cycloplegic agent used, duration of instillation, time gap from instillation of first drop to dilated refraction done and side effects of the drug if any. Results were analysed with respect to four parameters adherence to cycloplegic refraction (group-I), choice of cycloplegic agent (group-II), dosage of cycloplegia (group-III) and duration of cycloplegia (group-IV).

3) Standards: Since there are currently no standardized national guidelines or protocols for dilated refraction in children, we have established our own standard protocols in accordance with the available literature [3]-[5]; details mentioned in Table 1.

4) The audit cycle: A standard based audit of the dilatation practices among the pediatric optometrists and ophthalmologists was carried out.

a) First cycle: Case sheets of children in age group of 0 - 12 years with refractive errors and strabismus who visited pediatric ophthalmology outpatient services over a period of 4 weeks in the month of April 2012 were reviewed for the four parameters; this was compared to the hospital's dilatation protocol.

b) Intervention: The results of the first audit were analyzed and presented to the audit committee. All the case sheets where the choice of drug was not appropriate were re-evaluated and reasons for not complying with the protocol were noted. After full analysis of the first cycle, the dilatation protocol of the department was for- 
Table 1. Standardized protocol for dilation/cycloplegia ${ }^{3,4,5}$.

I All children younger than 12 years, with refractive error or strabismus should undergo cycloplegic refraction in the first visit Choice of cycloplegic agent

\begin{tabular}{|c|c|c|}
\hline Age & Category & Drug of choice \\
\hline$<2$ years & All children & Atropine (1\%) eye ointment \\
\hline \multirow{2}{*}{2 - 5 years } & With Esotropia [5] & Atropine (1\%) eye ointment \\
\hline & Without Esotropia [3] & Cyclopentolate (1\%)—Tropicacyl (1\%)—Cyclopentolate (1\%) (CTC) \\
\hline \multirow[b]{2}{*}{5 - 8 years } & With Esotropia [5] & Atropine (1\%) eye ointment \\
\hline & $\begin{array}{l}\text { Without Esotropia } \\
\text { Myopia [4] } \\
\text { Hyperopia [3] }\end{array}$ & $\begin{array}{c}\text { Tropicamide (1\%) eye drops } \\
\text { CTC }^{*}\end{array}$ \\
\hline \multirow{2}{*}{8 - 12 years } & With esotropia & СТC \\
\hline & Without Esotropia & Tropicamide (1\%) eye drops \\
\hline
\end{tabular}

Dosage of cycloplegic drugs

III - Atropine sulphate (1\%): eye ointment to be instilled twice a day for 3 days.

- Cyclopentolate (1\%) and Tropicamide (1\%) (CTC): 3 drops instilled at an interval of 15 minutes.

- Tropicamide (1\%): 3 drops instilled at an interval of 10 minutes each.

\section{Duration of cycloplegia}

IV Atropine sulphate (1\%): After 3 days of first instillation.

- Cyclopentolate (1\%) and Tropicamide (1\%) (CTC): After 60 minutes of first instillation.

- Tropicamide (1\%): Within 30 - 45 minutes of first instillation.

"CTC—Cyclopentolate (1\%)—Tropicacyl (1\%)—Cyclopentolate (1\%)—3 drops instilled at an interval of 15 minutes—refraction done after $60 \mathrm{mi}$ nutes of first installation.

matted into a poster, laminated and placed in all pediatric OPDs. All team members involved in the dilatation process were also educated about the appropriate drug, its dosage and duration of instillation.

c) Second cycle: All the case sheets of children younger than 12 years visiting pediatric ophthalmology outpatient services over a period of 4 weeks in the month of August 2012 were re-audited by the same therapist, for the same four parameters; results were assessed and published to the team.

5) Statistical analysis: Results of the two audit cycles were recorded and compared using SPSS 16 software. Chi-square test was used for comparison of values in each group in the first and second audit cycle; $p$-value of $\leq 0.05$ was considered significant.

\section{Results}

Demographic details of children in each cycle are summarized in Table 2. Comparison of values in each group in the first and the second audit cycle is summarized in Table 3.

First cycle: Of the 334 case sheets audited, 230 (69\%) were plain refractive errors and 104 (31\%) had associated strabismus. Cycloplegic refraction was performed in 275 (82.3\%) children while in the remaining 59 (17.7\%) glasses were prescribed based on dry refraction. Cycloplegic agent appropriate for age and category of patient was used for dilatation in $212(77 \%)$ cases. The duration and dosage of instillation was in accordance to formulated protocol in 231 (84\%) cases. Final refraction was done after three days in all the 44 children (100\%) of the atropine group; after 60 minutes of first drop in 65 out of 99 (65\%) children of the Cyclopentolate-Tropicamide-Cyclopentolate (CTC) group and within 30 - 45 minutes in 94 out of 131 cases of the Tropicamide group (Table 3).

In most of the cases where the formulated dilation protocol was not adhered to, no reason was documented on the case sheet for the same. In 16 case sheets, stickers were not available so as collect the data for refraction. Parents were not willing for dilated refraction or refraction with a particular cycloplegic agent in 6 cases. Other reasons included allergy to a particular drug, recent switch in department's protocol in a particular category of patients and an outstation patient where atropine refraction was not practical. 
Table 2. Demographic details.

\begin{tabular}{ccc}
\hline & First cycle & Second cycle \\
\hline Patients (n) & $\mathbf{3 3 4}$ & $\mathbf{4 3 6}$ \\
Age (years) (mean \pm SD) & $6.2 \pm 2.2$ & $7.0 \pm 2.9$ \\
$<$ 2 years & $32(9.6 \%)$ & $29(6.7 \%)$ \\
$\mathbf{2}$ - 5 years & $91(27.2 \%)$ & $104(23.8 \%)$ \\
$\mathbf{5}$ - 8 years & $120(40 \%)$ & $160(36.7 \%)$ \\
$\mathbf{8}$ - 12 years & $91(27.2 \%)$ & $143(32.8 \%)$ \\
Boys: Girls, Boys (\%) & $174 / 160,52 \%$ & $235 / 201,54 \%$ \\
\hline
\end{tabular}

Table 3. Comparison of the various protocols in first and second audit cycle.

\begin{tabular}{|c|c|c|c|c|}
\hline S no. & Criteria & First cycle n (\%) & Second cycle n (\%) & $p$-value \\
\hline 1 & Children included for audit & 334 (43.9\%) & 436 (51.9\%) & \\
\hline 2 & Dilated/Cycloplegic refraction done & 275/334 (82.3\%) & 411/436 (94.3\%) & 0.001 \\
\hline 3 & Appropriate Cycloplegia used & 212/275 (77\%) & 390/411 (94.8\%) & 0.001 \\
\hline 4 & Cycloplegia used in appropriate dosage & 231/275 (84\%) & 396/411 (96.3\%) & 0.001 \\
\hline 5 & $\begin{array}{l}\text { Refraction done after appropriate duration } \\
\text { - } \quad \text { Atropine } \\
\text { - } \quad \text { CTC } \\
\text { - } \text { Tropicamide }\end{array}$ & $\begin{array}{c}44 / 44(100 \%) \\
65 / 100(65 \%) \\
94 / 131(71.8 \%)\end{array}$ & $\begin{array}{c}42 / 42(100 \%) \\
158 / 162(97.5 \%) \\
203 / 207(98 \%)\end{array}$ & $\begin{array}{c}\text { Not applicable } \\
0.001 \\
0.001\end{array}$ \\
\hline
\end{tabular}

${ }^{*}$ CTC_Cyclopentolate (1\%) - Tropicacyl (1\%)—Cyclopentolate (1\%)—3 drops instilled at an interval of 15 minutesrefraction done after 60 minutes of first installation.

Second cycle: Of the 436 case sheets audited, 310 (71\%) were plain refractive errors and 126 (29\%) had associated strabismus. Cycloplegic refraction was performed in 411 (94.3\%) children while in the remaining 25 (5.7\%) glasses were prescribed based on dry refraction. Cycloplegic agent appropriate for age and category of patient was used for dilatation in 390 (94.8\%) cases. The duration and dosage of instillation was in accordance to formulated protocol in 396 (96.3\%) cases. Final refraction was done after 3 days in all the 36 children (100\%) of the atropine group, after 60 minutes of first drop in 158 out of 162 (97.5\%) cases of the CTC group and within 30 - 45 minutes in 203 out of 207 (98\%) children in the Tropicamide group.

\section{Discussion}

Evidence-based clinical guidelines, aiming to improve the effectiveness and efficiency of care delivery, have become a major feature of patient care [6] [7]. Non-adherence to guidelines may lead to unnecessary diagnostics, suboptimal treatment, or even adverse events [8] [9]. Owing to lack of knowledge of these standards or due a resistance in change of behaviour; some health care professionals and the technical staff may not adhere to the guidelines perfectly [10]. Active implementation through communication and education may improve adherence to guidelines and reduce sub-optimal care for patients [11].

In our study, each audit cycle provided demographically comparable populations of children with refractive error and strabismus. In the first audit cycle, poor adherence was observed in all the four study parameters. It was interesting to observe a statistically significant improvement in all these parameters in the second audit cycle. The parameters included were adherence to the dilatation protocol which improved markedly (82.3\% in first cycle to $94.3 \%$ in second cycle; $p=0.001)$, choice of cycloplegic agent $(77 \%$ in the first cycle to $94.8 \%$ in the second cycle, $p=0.001$ ), dosage of cycloplegic agent ( $84 \%$ in the first cycle to $96.3 \%$ in the second cycle, $p$ $=0.001$ ) and duration of cycloplegic agents ( $65 \%$ in the first cycle to $97.5 \%$ in the second cycle; $p=0.001$ for CTC and $71.8 \%$ in the first cycle to $98 \%$ in the second cycle; $p=0.001$ for Tropicamide) (Table 3 ).

The results of our study have several practical implications. Firstly, it indicates that despite availability of well defined standards, a greater number of children were prescribed inappropriate glasses. There is a possible explanation to this; junior medical staff might not understand the importance of appropriate drug, dosage and dura- 
tion of cycloplegia and could have been frequently unaware of the standard guidelines. Ours is a training institute with a huge turnover of optometrists performing pediatric refraction and fellow trainees in pediatric ophthalmology; this could account for the variations in practice. The second implication was finding a significant improvement in the practices following certain criteria aimed at increasing the awareness of the junior staff in the department. This included simple exercise like educating the staff and placing the aide memoires of the protocol at various places in the outpatient services.

Accurate prescription of glasses is the responsibility of the pediatric ophthalmology team. It was clear that compliance with guidelines was poor in all respects in the first audit cycle. This audit therefore targeted the practice patterns for cycloplegic refraction in children and the improvement clearly represents how simple measures can result in better adherence to protocols and uniformity in prescribing glasses. Though our results were encouraging, total compliance was not achieved with this audit. Achieving this must be the aim and re-auditing these results in the future after further re-enforcement of the guidelines would assess this.

Our study has certain limitations. The audit was dependent on clear documentation. Patients may have been assessed without documentation and therefore missed during data collection. However, the authors maintain that failing to document assessments is unsafe. Without documentation, the staff is left to pass on instructions, often causing confusion, and deteriorating examination findings. Another limitation was a relatively small number of children in either group; however statistical analysis demonstrated significant differences.

\section{Conclusion}

To conclude, our study demonstrated that a complete audit cycle with targeted interventions could be effective in improving compliance with evidence-based best clinical practice. Although predefined audit standard was not reached, the strategy used showed promise and further audit cycles would be needed to improve clinical practice further.

\section{References}

[1] Shah, P., Jacks, A.S. and Adams, G.G. (1997) Paediatric Cycloplegia: A New Approach. Eye, 11, 845-846. http://dx.doi.org/10.1038/eye.1997.216

[2] Mehta, A. (1999) Chief Complaint, History and Physical Examination. In: Santiago, A.P. and Rosenbaum, A.I., Eds., Clinical Strabismus Management, Saunders, Philadelphia, 18.

[3] Miranda, M.N. (1972) Residual Accommodation. A Comparison between Cyclopentolate 1 Percent and a Combination of Cyclopentolate 1 Percent and Tropicamide 1 Percent. Archives of Ophthalmology, 87, 515-517. http://dx.doi.org/10.1001/archopht.1972.01000020517004

[4] Manny, R.E., Hussein, M., Scheiman, M., et al. (2001) Tropicamide (1\%): An Effective Cycloplegic Agent for Myopic children. Investigative Ophthalmology \& Visual Science, 42, 1728-1735.

[5] Rosenbaum, A.L., Bateman, J.B., Bremer, D.L., et al. (1981) Cycloplegic Refraction in Esotropic Children. Cyclopentolate versus Atropine. Ophthalmology, 88, 1031-1034. http://dx.doi.org/10.1016/S0161-6420(81)80032-2

[6] (1992) Guidelines for Clinical Practice: From Development to Use. In: Lohr, K.N., Field, M.J., Eds., Institute of Medicine Committee on Clinical Practice Guidelines, National Academy Press, Washington DC,.

[7] Cabana, M.D., Rand, C.S., Powe, N.R., et al. (1999) Why Don’t Physicians Follow Clinical Practice Guidelines? A Framework for Improvement. JAMA, 282, 1458-1465. http://dx.doi.org/10.1001/jama.282.15.1458

[8] Foy, R., MacLennan, G., Grimshaw, J., et al. (2002) Attributes of Clinical Recommendations That Influence Change in Practice Following Audit and Feedback. Journal of Clinical Epidemiology, 55, 717-722. http://dx.doi.org/10.1016/S0895-4356(02)00403-1

[9] Grol, R. and Grimshaw, J. (2003) From Best Evidence to Best Practice: Effective Implementation of Change in Patients' Care. The Lancet, 362, 1225-30. http://dx.doi.org/10.1016/S0140-6736(03)14546-1

[10] Grol, R. and Buchan, H. (2006) Clinical Guidelines: What Can We Do to Increase Their Use? Medical Journal of Australia, 185, 301-302.

[11] Freemantle, N. (2000) Implementation Strategies. Family Practice, 17, S7-S10. http://dx.doi.org/10.1093/fampra/17.suppl_1.S7 
Scientific Research Publishing (SCIRP) is one of the largest Open Access journal publishers. It is currently publishing more than 200 open access, online, peer-reviewed journals covering a wide range of academic disciplines. SCIRP serves the worldwide academic communities and contributes to the progress and application of science with its publication.

Other selected journals from SCIRP are listed as below. Submit your manuscript to us via either submit@scirp.org or Online Submission Portal.
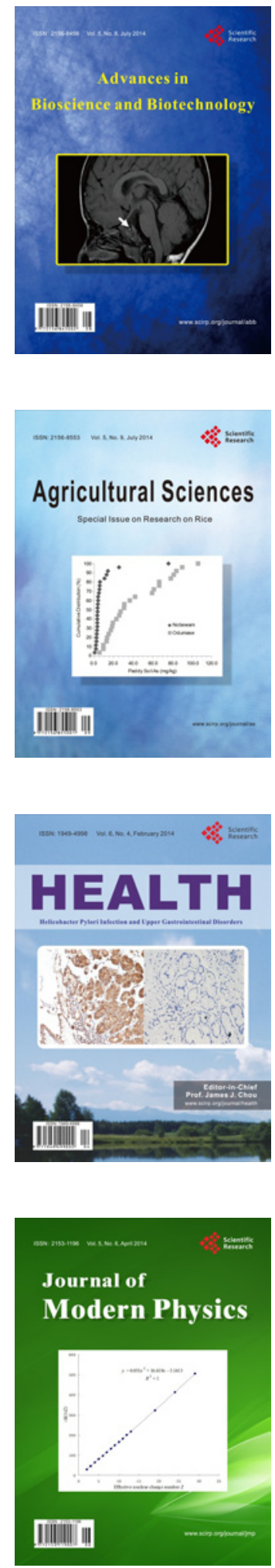
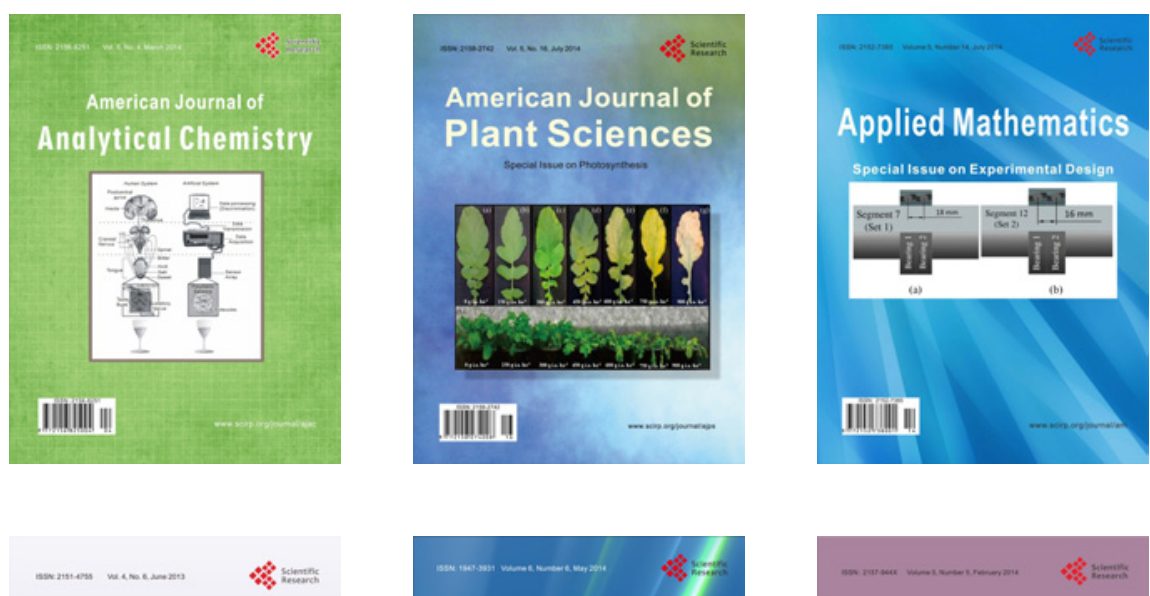

Creative Education
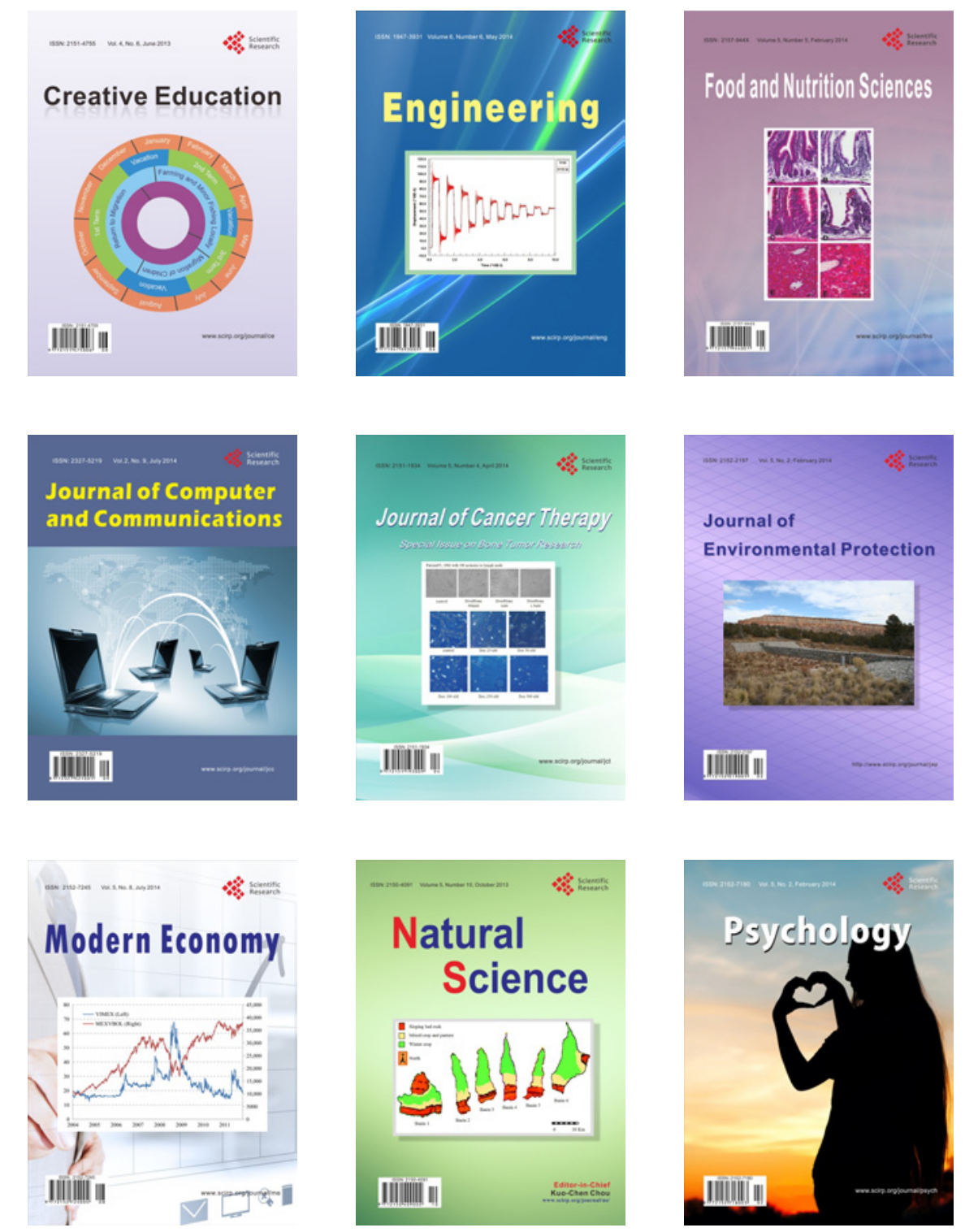